\title{
PN Jeffreys-Vicente Equation
}

\author{
Chongming $\mathrm{Xu}^{1,2}$, Xuejun $\mathrm{Wu}^{1,2}$ and Michael Soffel ${ }^{1}$ \\ ${ }^{1}$ Lohrmann Observatory, Technical University Dresden, \\ Germany ${ }^{2}$ Dept of physics, Nanjing Normal University, Nanjing \\ 210097, China
}

In our work (Xu et al. 2000) for the first time a complete and closed set of postNewtonian dynamical equations for elastically deformable, rotating astronomical bodies has been deduced. These bodies are assumed to differ only slightly from corresponding stationary and axisymmetric fluid reference bodies and show no internal dissipation, i.e., they are composed of a perfectly elastic medium. For such bodies a set of perturbation equations is derived by means of the Carter and Quintana formalism in a suitably defined rotating coordinate system. The post-Newtonian dynamical equation for the displacement field is the central result of this paper together with the various equations that define the quantities appearing therein. It is the post-Newtonian version of the well-known JeffreysVicente (J-V) equation (Jeffreys \& Vicente 1957) that forms the basis of any local theory of global geodynamics. $\mathrm{PN} \mathrm{J-V}$ equation reads:

$$
\begin{aligned}
0= & \rho^{*}\left(1+\frac{2 W_{\mathrm{G}}}{c^{2}}\right)\left(\ddot{s}_{a}+2 \epsilon_{a b c} \Omega^{b} \dot{s}^{c}\right)+\rho^{*} \Theta W_{\mathrm{G}, \mathrm{a}}-\rho^{*} s_{, a}^{b} W G_{\mathrm{G}, \mathrm{b}} \\
& -\rho^{*}\left(\delta W_{\mathrm{G}}\right)_{, a}-\rho^{*} s^{b} W_{\mathrm{G}, \mathrm{ba}}-\left(\kappa \Theta \delta_{a \beta}+2 \mu s_{a}^{\beta}\right)_{; \beta}+\frac{1}{c^{2}}\left\{\rho ^ { * } \left[V^{a}\left(V^{b} \ddot{s}^{b}\right)\right.\right. \\
& \left.+W_{\mathrm{G}, \mathrm{b}} \dot{s}^{b} V^{a}-2 V^{b} \dot{s}^{b} W_{\mathrm{G}, \mathrm{a}}+(\delta W)_{, t} V^{a}+8 \dot{s}^{b} W_{[b, a]}-4\left(\delta W_{a}\right)_{, t}\right] \\
& \left.+\kappa\left(\Theta W_{\mathrm{G}, \mathrm{a}}-\dot{\Theta} V^{a}\right)+\left(\kappa\left(4 W \Theta+V^{b} V^{c} s_{, c}^{b}\right)\right)_{, a}\right\}+\mathcal{O}(4)
\end{aligned}
$$

where $s_{a}$ is the covariant displacement field, $\rho^{*}=\rho+\frac{p}{c^{2}}$ ( $\rho$ is the density of tolal mass-energy, $p$ is the isotropic pressure), $W_{\mathrm{G}}$ a relativistic geopotential in rotating coordinate, $\Theta$ the volume dilation, $\delta W_{\mathrm{G}}$ Euler variation of $W_{\mathrm{G}}, \kappa$ the compression modulus, $s_{\alpha}^{\beta}$ is the shear tensor, $W$ and $W^{a}$ are the scalar and vector potential in rotating coordinate. Note that the part containing the shear-stress tensor reads explicitly

$$
\begin{aligned}
\left(2 \mu s_{a}^{\beta}\right)_{; \beta}= & \left(2 \mu s_{b a}\right)_{, b}+\frac{1}{c^{2}}\left\{-\left(4 \mu W s_{b a}+2 \mu s_{c a} V^{c} V^{b}\right)_{, b}+\left(2 \mu s_{a b}\right)_{, t} V^{b}\right. \\
& \left.+2 \mu\left(2 W_{, c} s_{a c}+\epsilon_{a c b} \Omega^{c} s_{b d} V^{d}\right)\right\} .
\end{aligned}
$$

This is the desired dynamical equation for the displacement field. It is valid up to terms of $\mathcal{O}(4)$ and second order in the displacement field itself. When $1 / c^{2}$ parts are omitted, the equations just return back to $\mathrm{J}-\mathrm{V}$ equation. The extended PN J-V equation is the first time obtained by us. 


\section{References}

Xu, C., Wu, X. \& Soffel, M. 2000, submitted to Phys. Rev. D

Jeffreys, H. \& Vicente, R.O. 1957, Mon. Not. R. Astro. Soc., 117, 142 